\title{
Pistachio and cashew nut allergy in childhood: Predictive factors towards development of a decision tree
}

\author{
Pinar Gur Cetinkaya, ${ }^{1}$ Dilara Karaguzel, ${ }^{2}$ Saliha Esenboğa, ${ }^{1}$ Umit Murat Sahiner, ${ }^{1}$ Ozge Soyer, ${ }^{1}$ Betul Buyuktiryaki, ${ }^{1}$ \\ Esra Birben, ${ }^{1}$ Çagatay Karaaslan, ${ }^{2}$ Bulent Enis Sekerel ${ }^{1}$
}

\begin{abstract}
Background: Pistachio and cashew nut, which belong to the same botanical family, are tree nuts that induce serious allergic reactions.

Objective: We aimed to determine the predictive factors for pistachio and cashew nut reactivity during oral food challenge (OFC).

Methods: A total of 112 pistachio and/or cashew nut sensitized children, aged 58.45 (IQR:40.38-88.32) months, were included. Cutoff values and probability curves for skin prick test (SPT), sIgE, sIgE/Total IgE that predict reactivity were determined for pistachio and cashew nut. Additionally, a diagram was created that can be useful while making a decision for OFC based on SPT and SIgE values.

Results: A total of 73 patients underwent OFC with pistachio and/or cashew nut. Twelve children with current anaphylaxis history were not challenged and accepted as allergic. SPT was the only predictive factor for positive pistachio/ cashew nut OFC. According to area under curve (AUC) analysis, SPT was more predictive than sIgE and sIgE/Total IgE both for pistachio and cashew nut. Optimal cutoff values according to "Youden index" for pistachio SPT, sIgE, and sIgE/Total IgE were $7.25 \mathrm{~mm}, 4.14 \mathrm{kUA} / \mathrm{L}$, and $1.32 \%$, respectively. And those values for cashew nut SPT, sIgE, and sIgE/Total IgE were $6.25 \mathrm{~mm}, 1.125 \mathrm{kUA} / \mathrm{L}$, and $3.30 \%$, respectively. The diagram showed that SPT predicted the reactivity together with sIgE better than only the SPT values.
\end{abstract}

Conclusion: SPT was the best predictor for reactivity both for pistachio and cashew nut. Combined use of SPT and sIgE may improve the prediction of reactivity at pistachio and cashew nut OFCs in children.

Key words: Cashew nut, cutoff value, oral food challenge, pistachio, probability curve

\section{From:}

Division of Pediatric Allergy and Asthma Unit, Department of Pediatrics, Faculty of Medicine, Hacettepe University, 06100 Sihhiye, Ankara, Turkey

${ }^{2}$ Department of Biology, Faculty of Science, Hacettepe University, 06800 Ankara, Turkey

\section{Introduction}

Tree nuts can lead to life-threatening allergic reactions and are among the foods most commonly causing anaphylaxis. ${ }^{1}$ Tree nut allergy and consumption vary considerably based on geographical regions and cultural traditions. ${ }^{2}$ Turkey is one of the major pistachio cultivating and consuming regions in the world. ${ }^{3}$ Because of these facts, allergy to pistachio is predicted to occur more commonly in Turkey than in many other countries. Because of pistachio and cashew nut belong to the botanically same family Anacardiaceae, cashew nut allergy is also observed frequently in patients with pistachio allergy. ${ }^{4}$

\author{
Corresponding author: \\ Bulent Enis Sekerel \\ Hacettepe University School of Medicine, \\ Department of Pediatric Allergy, 06100 Ankara, Turkey \\ E-mail: b_sekerel@yahoo.com
}

Oral food challenge (OFC) (particularly the double-blind placebo-controlled food challenge) tests are the gold standard method to diagnose the clinical reactivity. ${ }^{5}$ Unfortunately, severe allergic reactions may occur during OFCs. ${ }^{6}$ If the laboratory and clinical parameters predicting the clinical reactivity can be determined, the possibility of severe reactions may diminish, and unnecessary avoidance may be prevented. Although clinical and laboratory studies have been reported about nut allergy, ${ }^{7,8}$ a clear consensus on the laboratory and/or clinical predictors for the reactivity have not been determined yet. 
In this study, we aimed to reveal laboratory parameters that can predict the reaction, because of pistachio and cashew nut oral provocation test results in serious allergic reactions.

\section{Methods \\ Study population}

This prospective study was conducted at the Division of Pediatric Allergy in Hacettepe University from July 1, 2015, to June 30, 2017. All children (0-18 years old) who had sensitization to pistachio (sIgE or SPT) and/or cashew nut with or without any history of previous allergic reactions to these nuts were recruited into the study. The study protocol has been approved by the local ethical committee, and the parents/guardians gave written informed consent (Hacettepe University, GO 15/649-07). Data including age, gender, and initial and subsequent reactions to pistachio and cashew nut, atopic dermatitis $(\mathrm{AD})$ at any time during their lives ( $\mathrm{AD}$ ever) or $\mathrm{AD}$ in the previous 12 months (current $\mathrm{AD}$ ), presence of asthma and allergic rhinitis, food allergy, and family history of atopy were collected from patients' files and by asking the parents/guardians during the visits. Diagnoses of $\mathrm{AD}$, asthma, and allergic rhinitis were made according to international guidelines, ${ }^{5,9-13}$ Diagnosis of pistachio and cashew nut allergies were based on positive OFC and/or a clear-cut history of anaphylactic reactions within the previous 12 months with positive SPT and/or specific immunoglobulin E (sIgE) levels for pistachio/cashew nut. ${ }^{14}$ Reactions during OFC and previous history of allergic reactions (urticaria, angioedema, or anaphylaxis) to pistachio and/or cashew nut were graded based on international anaphylaxis guidelines. ${ }^{15}$

\section{Diagnosis of pistachio and cashew nut sensitization}

SPT with cashew nut and pistachio extracts was performed and on all participants by the same trained team who were proficient on SPT. Extracts for SPT were prepared from raw pistachio and cashew nut according to the method given below. The prick test result was considered positive if the mean wheal diameter was $3 \mathrm{~mm}$ greater than the negative control.

Total and sIgE levels were measured via the Pharmacia CAP system (Immunocap-CAP; Pharmacia \& Upjohn, Uppsala, Sweden); sIgE levels $\geq 0.35 \mathrm{kUA} / \mathrm{L}$ were accepted as positive for pistachio/cashew nut.

\section{Preparation of pistachio and cashew nut extracts}

Cashew nut and pistachio protein extracts were obtained from de-fatted raw powder by extraction with a buffer solution [ $\mathrm{PH} 7.4,0.5$ gr NaCl, 0.036 gr $\mathrm{KH}_{2} \mathrm{PO}_{4}$ (monobasic potasium phosphate), 0.764 gr $\mathrm{Na}_{2} \mathrm{HPO}_{4} \cdot 2 \mathrm{H}_{2} \mathrm{O}$ (dibasic sodium phosphate), 0.4 gr crystalized phenol in $100 \mathrm{ml}$ distilled water] followed by shaking for 4 hours at room temperature. The supernatant of the mixture obtained after centrifugation was stored at $-80^{\circ} \mathrm{C}$ for use during analysis.

\section{Oral food challenges}

Pistachio and cashew nut OFCs were performed in an open manner based on the international guidelines. ${ }^{16,17}$ Pistachio and cashew nut were introduced in roasted form to the patients during OFC. The test was stopped and accepted positive after the occurrence of objective symptoms.

\section{Statistical analysis}

Statistical analyses were performed using SPSS Version 22.0 statistical software package (IBM SPSS Statistics Chicago, Ill). Categorical values were not normally distributed; thus, the data are given as median and interquartile ranges (IQR). Odds ratios (ORs) with relevant 95\% confidence intervals (CIs) were calculated by univariate and multivariate analyses to predict the potential associations between pistachio and cashew nut SPT, sIgE, and sIgE/Total IgE measurements and oral provocation positivity to these nuts. Variables were selected if the $p$ value was less than 0.20 in the univariate analysis and included in multivariate analysis. A p-value of less than 0.05 was considered as the risk factors in multivariate analysis.

The accuracy of clinical reactivity to pistachio and cashew nut was determined using receiver operating characteristics (ROC) curves by analyzing with SPT $(\mathrm{mm}), \operatorname{sIgE}(\mathrm{kUA} / \mathrm{L})$, and $\operatorname{sigE}(\mathrm{kUA} / \mathrm{L}) /$ Total $\operatorname{IgE}(\mathrm{kU} / \mathrm{L})$ measurements. Positive predictive values (PPV), negative predictive values (NPV), sensitivity, specificity, positive likelihood ratios (sensitivity/1-specificity), and negative likelihood ratios (1-sensitivity/ specificity) were calculated using two-by-two tables for cutoff points. The optimal cutoff value for each variable was selected according to the Youden index (sensitivity + specificity -1). ROC curve analyses were performed in patients who had positive OFCs or anaphylaxis to pistachio or cashew nut within the previous 12 months. Predicted probability curves were generated by using logistic regression models for both pistachio and cashew nut.

\section{Results}

A total of 112 children (M/F: 74/38) with a median age of 58.45 (IQR: 40.38-88.32) months were enrolled. Median age of reaction history was 14.00 (12.00-22.50) months and 24.00 (17.75-48.00) months for pistachio and cashew nut, respectively. Sixty-eight $(60.7 \%)$ patients had a history of allergic reactions after consumption of pistachio and/or cashew nut, and the remaining patients had positive sIgE and/or positive SPT for pistachio and/or cashew nut. Fifty-eight of the 68 patients had positive clinical history to pistachio, while 30 had a positive clinical history to cashew nut. Almost 70.5\% had ever AD and $54.5 \%$ had current $\mathrm{AD}$. Moreover, $40.2 \%, 17 \%$, and $60 \%$ of the children had asthma, allergic rhinitis, and food allergy (other than tree nuts and peanut), respectively.

Skin was the most commonly affected organ based on clinical history [pistachio $(\mathrm{n}=51,88 \%)$, cashew nut $(\mathrm{n}=27$, $90 \%)$, and also in OFCs and in history of anaphylaxis within the last 12 months [pistachio (91\%), cashew nut (90.5\%)]. This was followed by lower respiratory tract $(32.8 \%)$, gastrointestinal system (20.7\%), upper respiratory tract (15.5\%), cardiovascular system (9\%) involvements, and patients with conjunctival injections (4.8\%); however, there was no patient with neurological system involvement for pistachio based on clinical history. Moreover, upper respiratory tract (43\%), gastrointestinal (43\%), and lower respiratory system (33.7\%) were the involved systems for pistachio allergic children based on OFCs and in history of anaphylaxis within the last 12 months, respectively. Nevertheless, there was neither cardiovascular nor neurological system involvement in this group. On the other hand, gastrointestinal system (26.7\%), 
upper respiratory $(26.7 \%)$, and lower respiratory tracts (20\%) were the other frequently observed systems in patients with reactions to cashew nut according to clinical-based history. This was followed by the patients with cardiovascular system symptoms (6.7\%), conjunctival injection (3.3\%), and neurological system symptoms (3.3\%). For the OFCs/anaphylaxis (within the last 12 months)-based history, involved systems were gastrointestinal (54.5\%), upper respiratory $(45.5 \%)$ and lower respiratory tracts (36.4\%), cardiovascular (9\%), and neurological $(3.2 \%)$ along with the symptom of conjunctival injection (9\%), respectively. Grading of the allergic reactions were done according to the international guidelines. ${ }^{15}$ Grade 1 and 2 allergic reactions were more common with pistachio in patients who had performed OFC and patients with previous accidental reactions. Otherwise, grade 3 and 4 reactions were more common in those patients with cashew nut.

\section{Characteristics of OFCs in the study population}

In the study population, 109 and 104 patients sensitized to pistachio and cashew nut, respectively, according to SPT and/ or sIgE values. Additionally, 103 of the study group sensitized to both pistachio and cashew nut with regard to SPT and/or sIgE. OFCs were performed on 71 children for pistachio and 48 children for cashew nut. Twelve patients had anaphylaxis with pistachio and/or cashew nut within the last 12 months and were accepted as allergic without OFC.

Ten out of 71 pistachio OFCs resulted in reaction, and 3 of them were anaphylaxis (30\%). On the other hand, 9 of 48 cashew nut OFCs were positive, and 7 of those reactions resulted in anaphylaxis (77.8\%). Only one child had OFC positivity with both pistachio and cashew nut. In total, $14 \%$ of OFC with pistachio and $18.7 \%$ of OFC with cashew nut were positive.

Children who had previous anaphylaxis with pistachio and cashew nut resulted in a much more positive reaction during OFCs and anaphylaxis after accidental consumption than children who did not have a previous history with these nuts (pistachio, $\mathrm{p}=0.032$; cashew nut, $\mathrm{p}=0.015)$.

In summary, 21 (19.2\%) (10 of them had positive OFC results with pistachio, and 11 of them had anaphylaxis with the pistachio in the last 12 months) of 109 pistachio-sensitized children were identified as allergic to pistachio. Thirteen (12.5\%) (9 of them had the positive OFC with cashew nut, and 4 of them had anaphylaxis with cashew nut in the last 12 months) of 104 cashew nut-sensitized children were identified as allergic to cashew nut. In addition, 3 of 21 (14.2\%) children with pistachio allergy reacted to cashew nut, and 3 of 13 $(23 \%)$ children with cashew nut allergy reacted to pistachio.

\section{Differences between reactive and non-reactive patients}

In children reactive to pistachio and cashew nut, the median levels of sIgE, SPT diameters, and sIgE/Total IgE ratios were significantly higher than those of the non-reactive subjects (Table 1).

Among the laboratory and clinical parameters, gender, SPT wheal size, sIgE/Total IgE ratio in percent, anaphylaxis after accidential consumption in history, and allergic rhinitis appeared to correlate with pistachio anaphylaxis in univariate analysis. Furthermore, gender, SPT wheal size, sIge values,
sIgE/Total IgE ratio in percent, $\mathrm{AD}$, and pollen sensitization were the significant parameters related with cashew nut anaphylaxis identified in univariate analysis. However, only the SPT wheal size was determined to be a predictive factor for estimating clinical reactivity in multivariate analysis [OR: 1.272, 95\% CI: $1.058-1.529, \mathrm{p}=0.011$ for pistachio] [OR: $1.308,95 \%$ CI: $1.057-1.619, \mathrm{p}=0.014$ for cashew nut].

\section{Cutoff values and predicted probability curves for pistachio and cashew nut sIgE, SPT, and sIgE/Total IgE ratio to predict clinical reactivity}

ROC curve analysis was performed to patients who underwent OFC with cashew nut and/or pistachio or who had anaphylaxis in the last 12 months to estimate the diagnostic accuracy of sIgE values, SPT wheal sizes, and sIgE/Total IgE ratios of pistachio and cashew nut to make a distinction between allergy and tolerance (Figure 1a and 1b). SPT wheal diameters for pistachio (area under the curve $(\mathrm{AUC})=0.845, \mathrm{p}<0.001$ ) (Figure 1a) and cashew nut $(\mathrm{AUC}=0.901, \mathrm{p}<0.001)$ (Figure 1b) were the most accurate predictors for predicting allergy. Additionally, sIgE/Total IgE ratio was the second, and $\operatorname{sgE}$ values alone was the third valuable parameters for both pistachio and cashew nut allergy according to ROC curve analysis (Figure 1a and 1b). Estimated optimal cutoff values of SPT, sIgE, and sIgE/Total IgE ratio were $7.25 \mathrm{~mm}, 4.14 \mathrm{kUA} / \mathrm{L}$, and $1.32 \%$ for pistachio, and $6.25 \mathrm{~mm}, 1.125 \mathrm{kUA} / \mathrm{L}$, and $3.30 \%$ for cashew nut. Sensitivity, specificity, PPV, and NPV values were defined for pistachio and cashew nut SPT, sIgE and sIgE/ Total IgE ratio. However, the results were the best for SPT diameters of pistachio.

The probability curves were generated at each value of pistachio and cashew nut SPT diameters, sIgE and sIgE/Total IgE ratios based on a logistic regression model (Figure 2a2f). However, 95\% probability of clinical reactivity could not be calculated for sIgE and sIgE/Total IgE values for pistachio, even by using log-transformed data. SPT levels predicted the clinical reactivity at $50 \%$ probability were $11.2 \mathrm{~mm}$ for pistachio (Figure 2a) and $11.25 \mathrm{~mm}$ for cashew nut (Figure 2d), at $95 \%$ probability were $21.2 \mathrm{~mm}$ for pistachio (Figure 2a) and $21.25 \mathrm{~mm}$ for cashew nut (Figure 2d).

We generated a diagram resembling a decision tree by the combination of both SPT and sIgE cutoff values to obtain more precise results predicting the reactivity with pistachio and cashew nut to help deciding the time of OFC (Figure $\mathbf{3 a}$ and $\mathbf{3 b}$ ). The values given in the decision tree represent a range rather than point values. SPT was more valuable in clinical practice according to our results; however, combination of SPT along with sIgE measurements had a higher clinical value rather than applying SPT alone to predict the tolerance both for pistachio and cashew nut. In addition, we further analyzed the combined SPT and sIgE levels below the cutoff values, and at that point sensitivity and NPV decreased, specificity and PPV increased while predicting the reactivity as well as the tolerance for pistachio and cashew nut (Table 2). Moreover, positive LRs were considerably increased compared to the individually analyzed positive LRs for SPT and SIgE. The frequency and the risk of the patients with pistachio and cashew nut reactivity increased by using both SPT and sIgE levels above the cutoff values than by using only SPT measurement. 


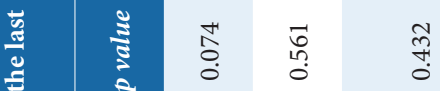

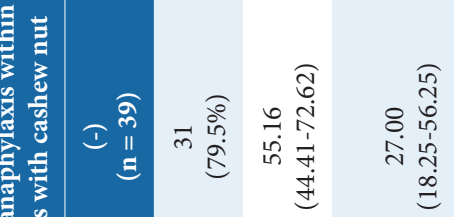

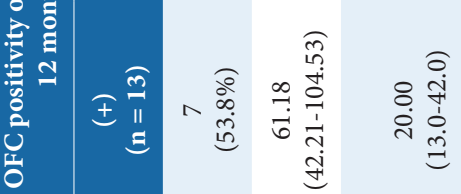

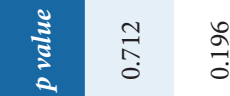

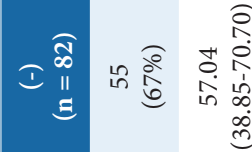

竞



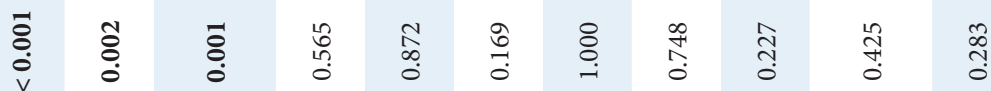

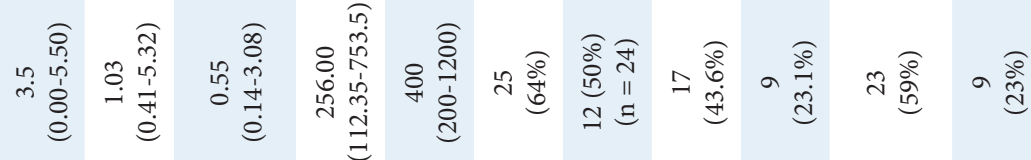

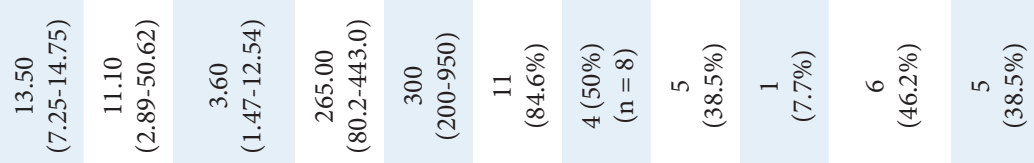

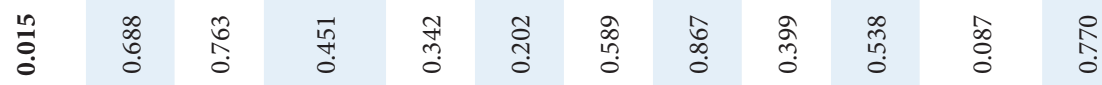

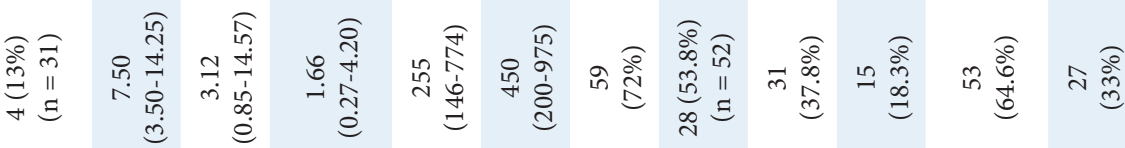



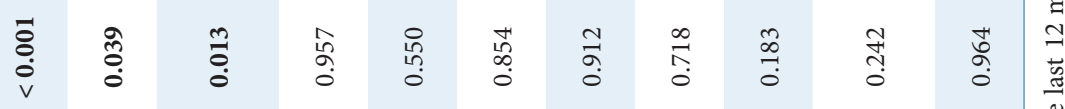

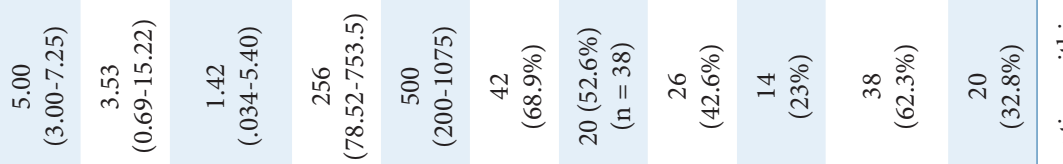



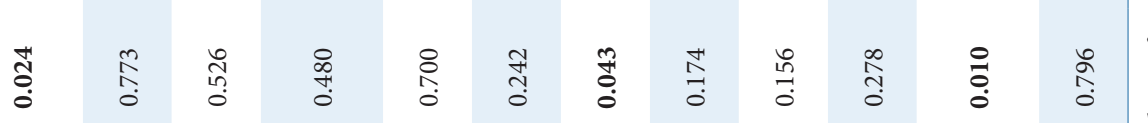

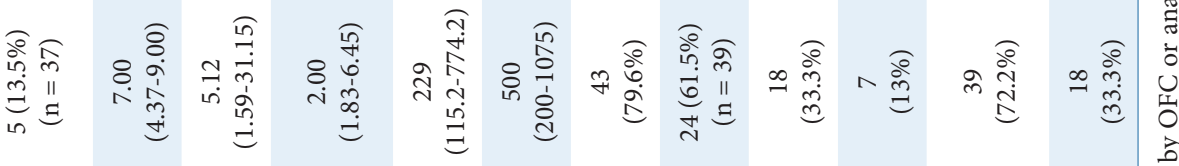

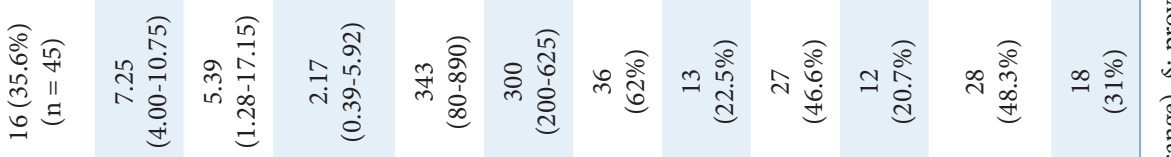

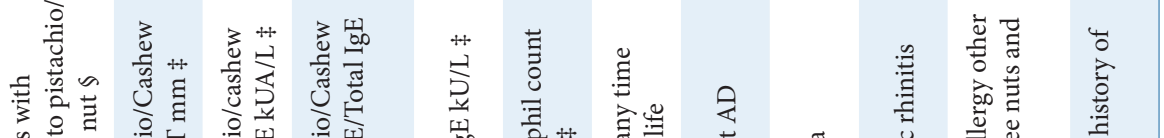

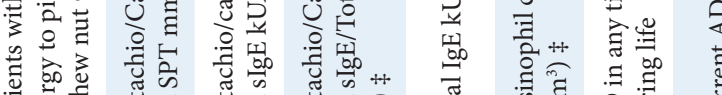

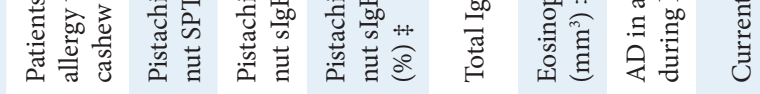




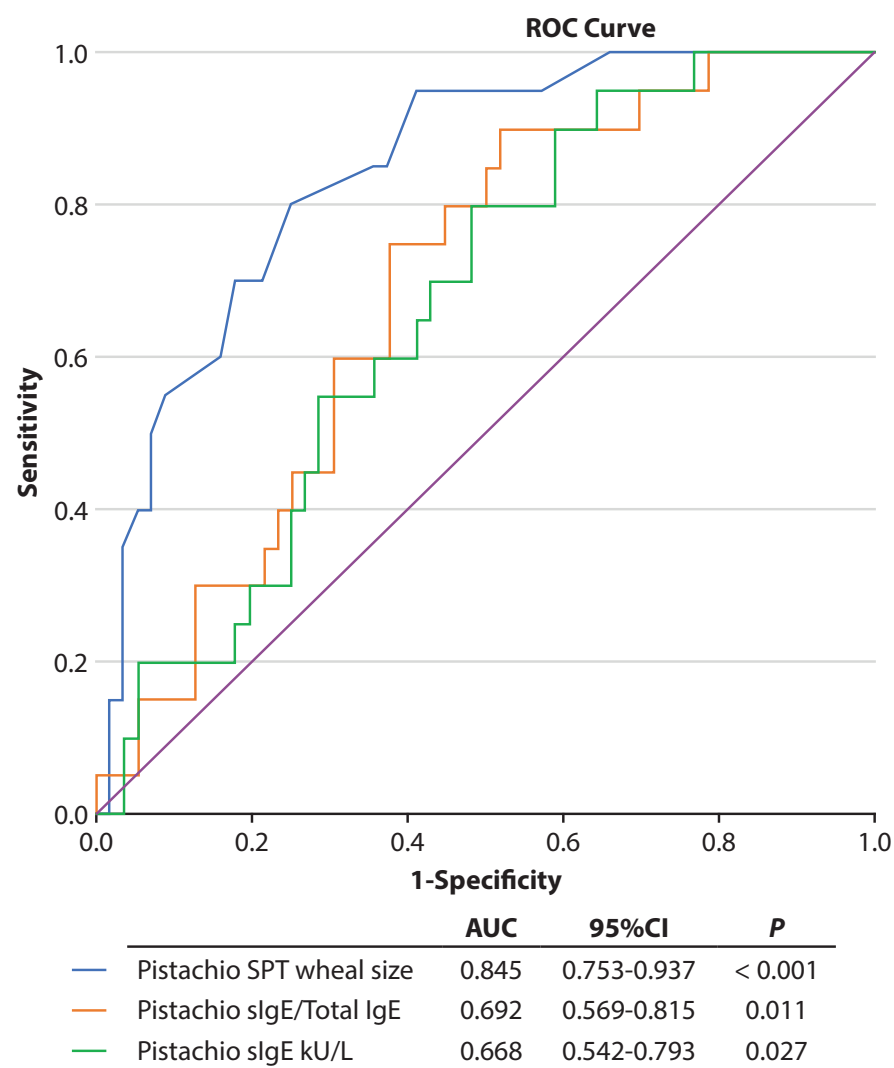

Figure 1a. Receiver operating characteristics (ROC) Curve for Pistachio

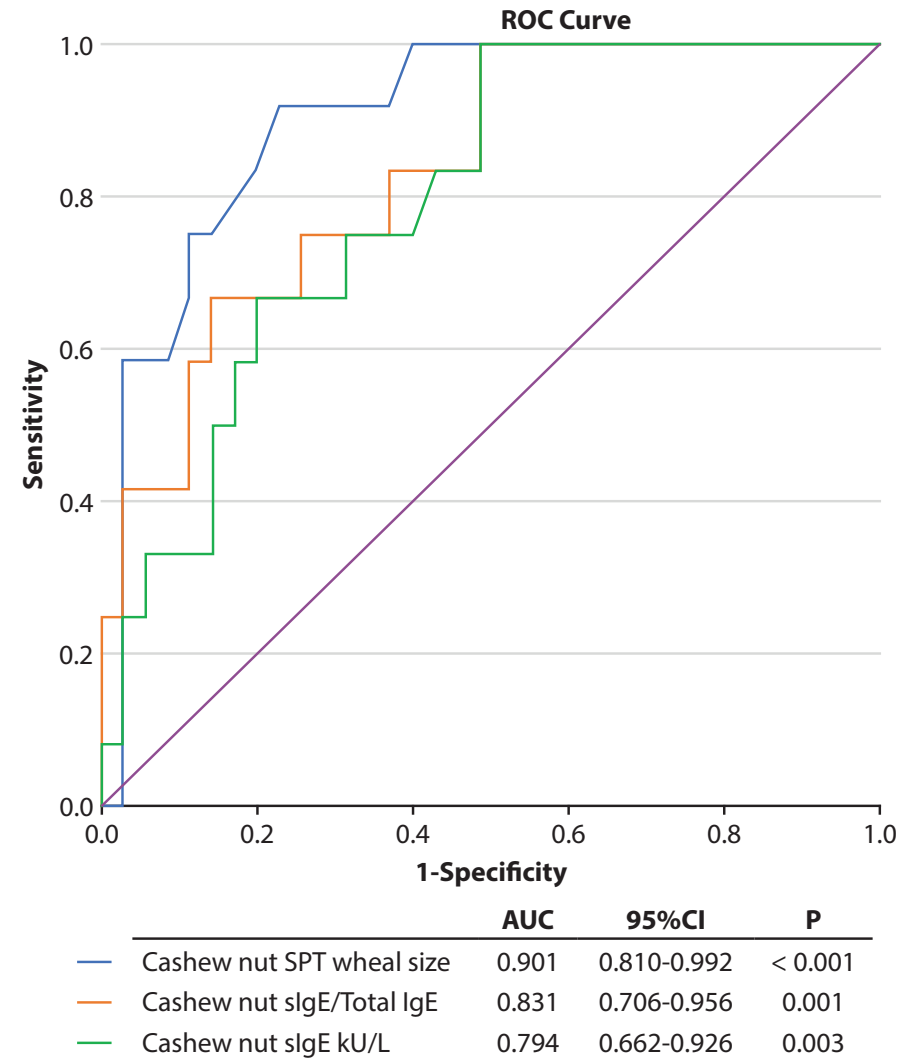

Figure 1b. Receiver operating characteristics (ROC) Curve for Cashew nut

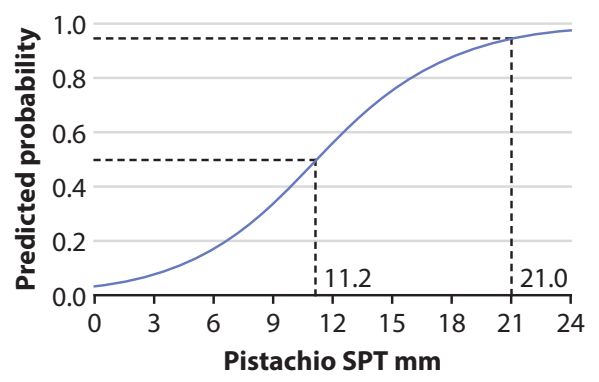

Figure 2a. Probability curve for clinical reactivity to pistachio at a given SPT wheal size

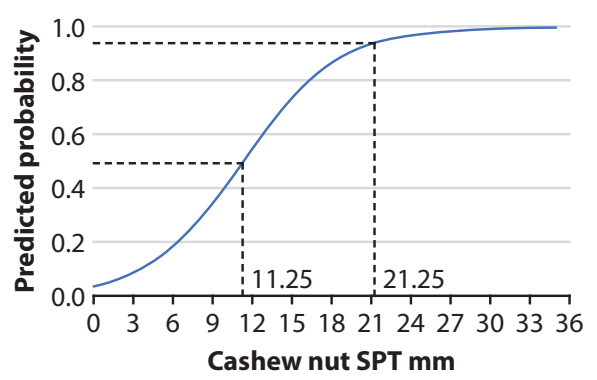

Figure 2d. Probability curve for clinical reactivity to cashew nut at aiven SPT wheal size

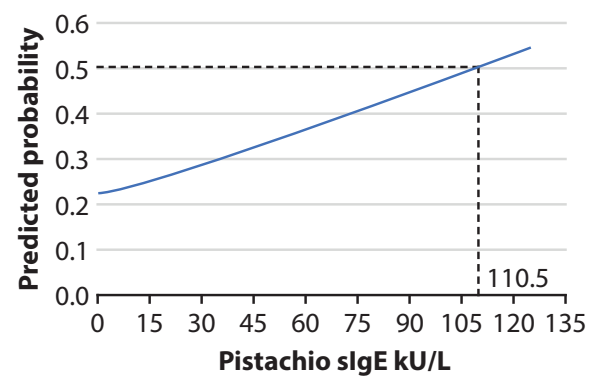

Figure 2b. Probability curve for clinical reactivity to pistachio at a given sIgE value

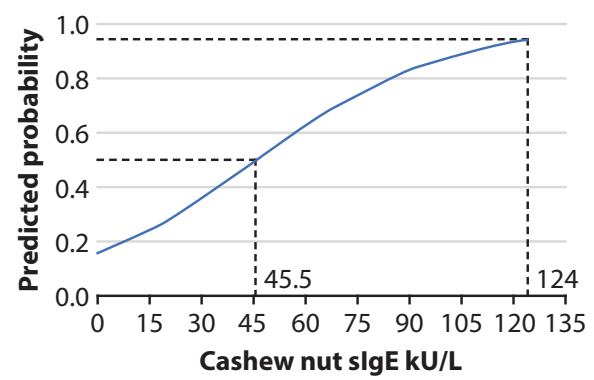

Figure 2e. Probability curve for clinical reactivity to cashew nut at given sIgE value

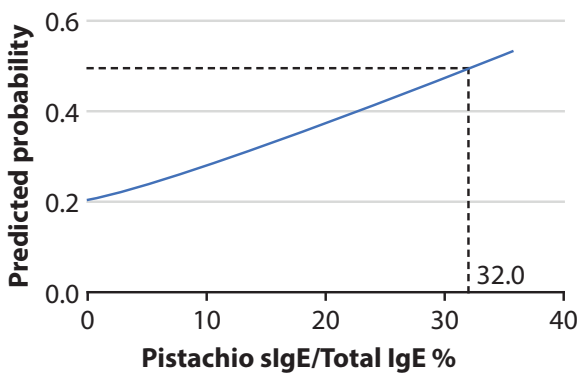

Figure 2c. Probability curve for clinical reactivity to pistachio at a given sIgE/Total IgE ratio

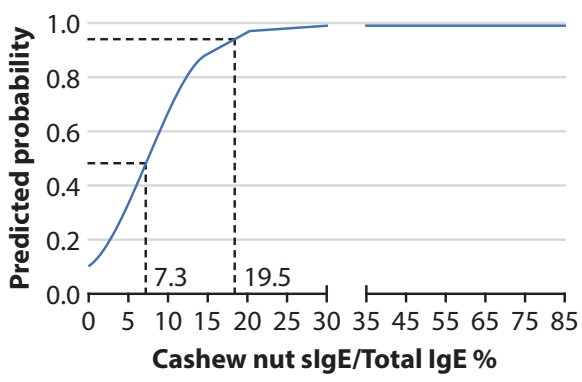

Figure 2f. Probability curve for clinical reactivity to cashew nut at aiven sIgE/Total IgE ratio 


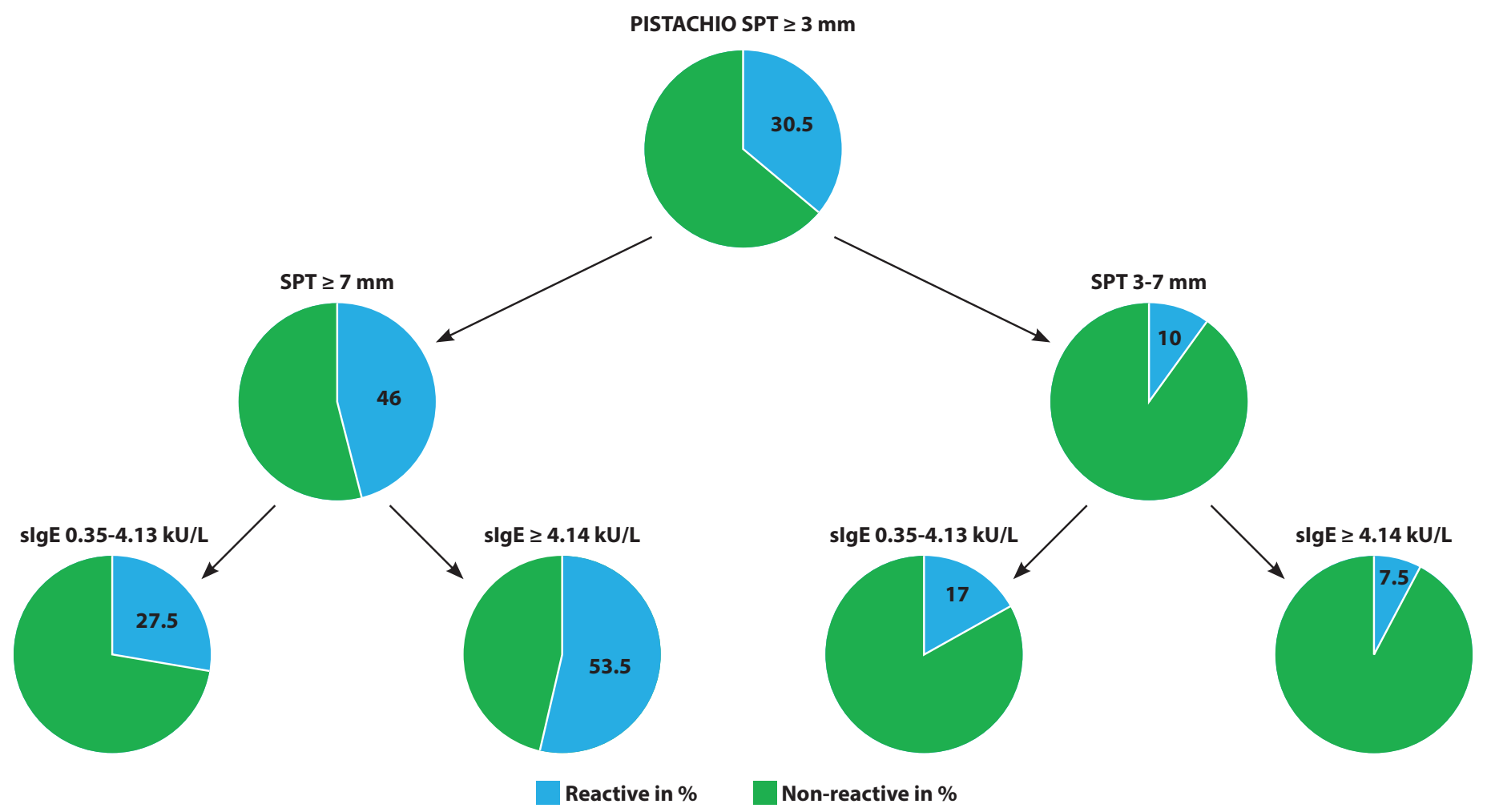

Figure 3a. The diagram of the study population showing increase in the prediction of reactive patients in the combination of SPT and sIgE levels above cutoff values for patients with pistachio allergy

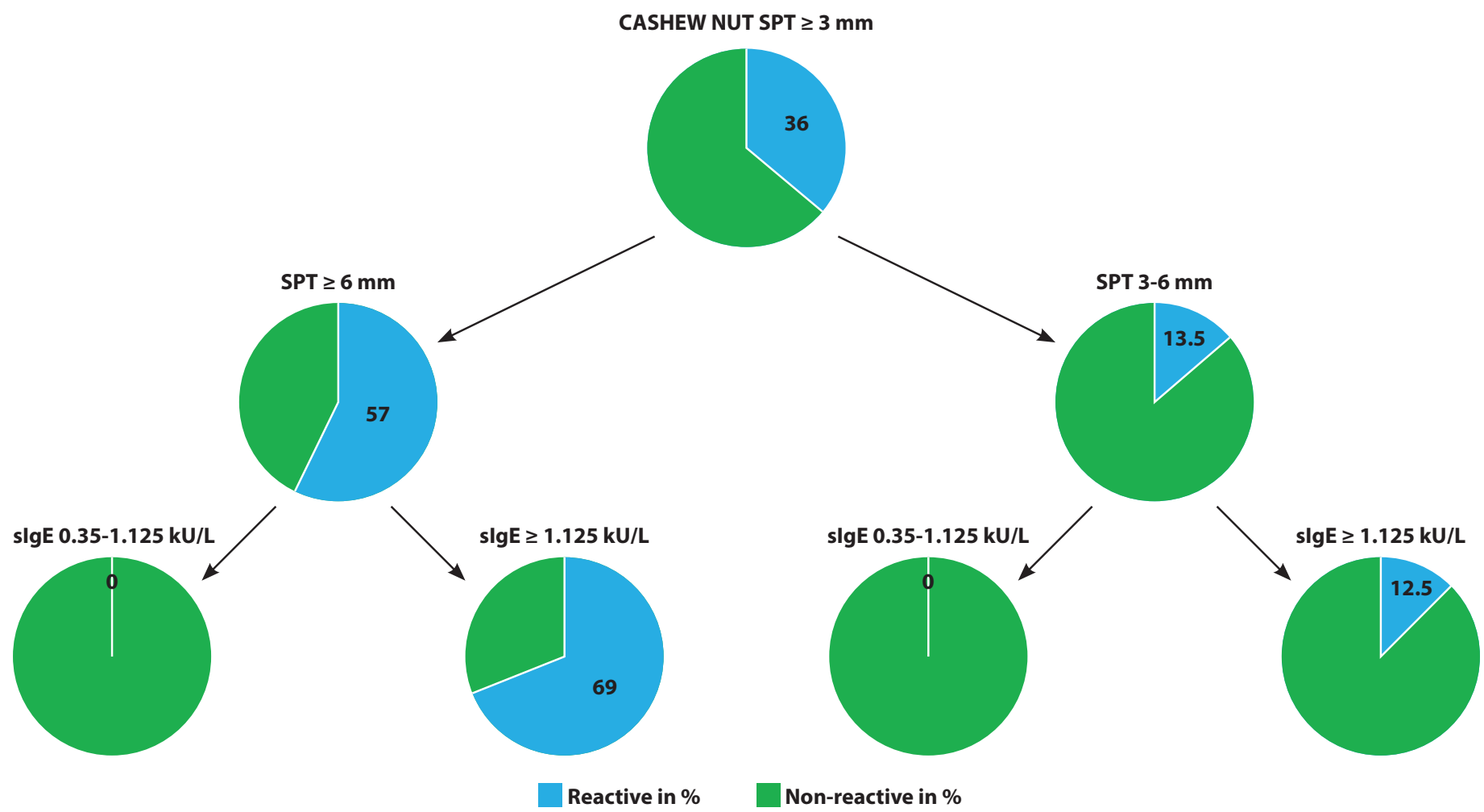

Figure 3b. The diagram of the study population showing increase in the prediction of reactive patients in the combination of SPT and sIgE levels above cutoff values for patients with cashew nut allergy 
Table 2. The use of combined SPT and sIgE cutoff values both for pistachio and cashew nut to predict the clinical reactivity with these two nuts

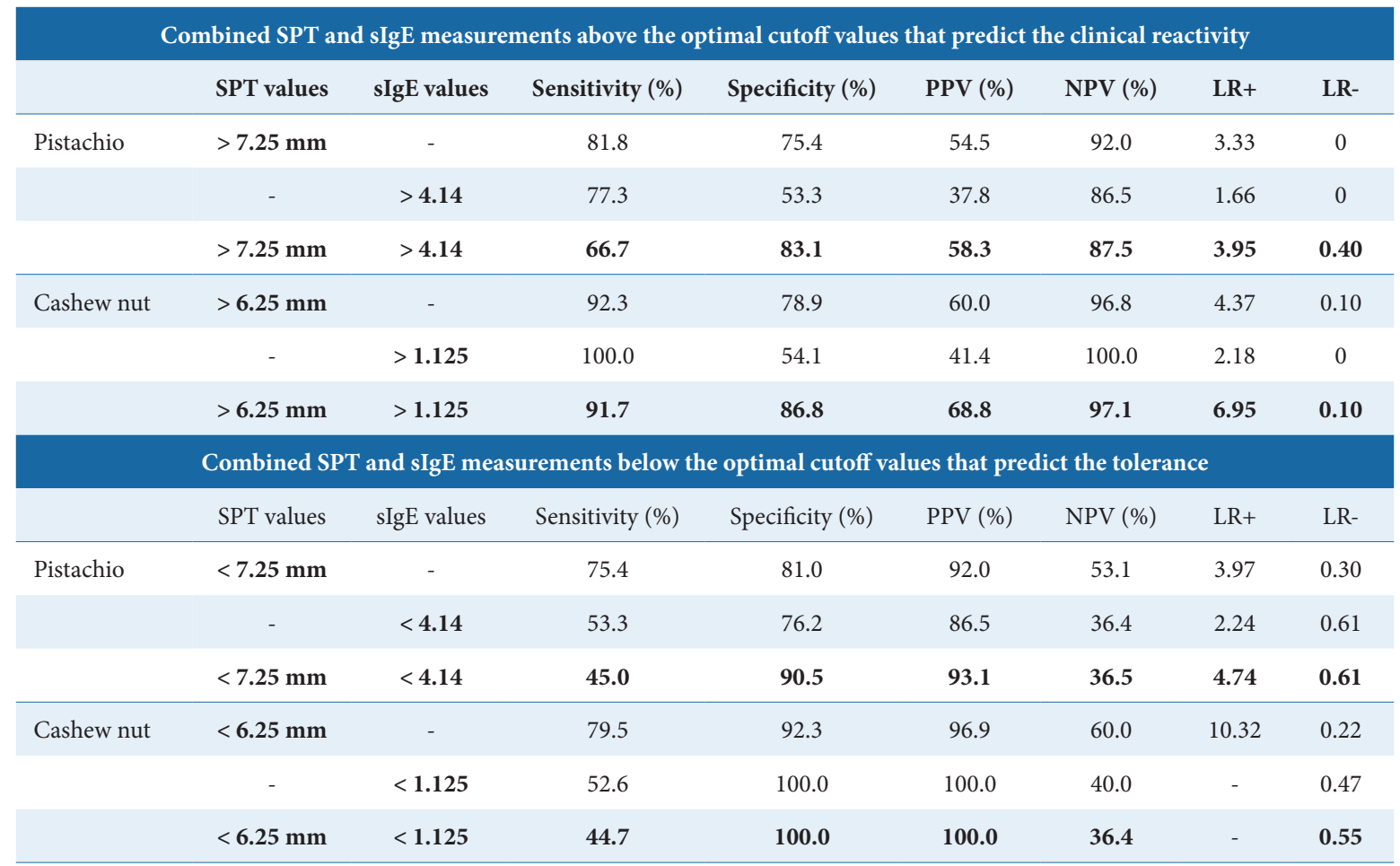

PPV; Positive Predictive Value, NPV; Negative Predictive Value, LR+; positive Likelihood Ratio, LR-; negative Likelihood Ratio

Therefore, we suggested using SPT together with sIgE to make a more accurate OFC decision.

\section{Discussion}

Results showed that SPT wheal size both for pistachio and cashew nut was the best indicator for predicting reactivity in provocation tests. In addition, sIgE/Total IgE ratio for these two nuts was a better predictor of OFC positivity than $\operatorname{sgE}$ values. Optimal cutoff values and probability curves for SPT wheal sizes, sIgE values, and sIgE/Total IgE ratio anticipating clinical reactivity were created for pistachio and cashew nut. The diagram created for estimation the appropriate time for OFC with pistachio and cashew nut suggested that combination of sIgE with SPT values had a higher possibility of reactivity.

SPT wheal size was the most, and $\operatorname{sIgE}$ was the second valuable predictors for predicting the reactivity to both pistachio and cashew nut in the current study. All these outcomes are consistent with previous literature results. ${ }^{7,18,19}$

In the current study, because only SPT and sIgE positivity are not sufficient to determine the reactive patients, ${ }^{20}$ we aimed to find optimal cutoff values for sIgE, SPT, and sIgE/ Total IgE ratio that estimated the risk of reaction during OFC with cashew nut and pistachio that have been reported previously. ${ }^{7,821}$ Ho et al. found a cashew nut SPT threshold of $\geq 8 \mathrm{~mm}$ that predicted the positivity in OFC with a > 95\% accuracy. ${ }^{22}$ However, $>95 \%$ accuracy was not achieved for pistachio in that study. ${ }^{22}$ Ludman et al. determined $\operatorname{sigE}$ for cashew nut and other tree nuts as $\geq 5 \mathrm{kUA} / \mathrm{L}$ in children with a $67 \%$ positive OFC prediction. ${ }^{21}$ In another report, the negative predicted value of $50 \%$ for cashew nut was 6.5 $\mathrm{mm} .^{7}$ Inoue et al found positive predictive values for cashew nut sIgE as $12.8 \mathrm{kUA} / \mathrm{L}$ with $50 \% \mathrm{PPV}$ and $149.5 \mathrm{kUA} / \mathrm{L}$ with 95\% $\mathrm{PPV}^{23}$ We found the threshold value for positive OFC with cashew nut as $6.25 \mathrm{~mm}$ for SPT, similar to previous studies. However, $1.12 \mathrm{kUA} / \mathrm{L}$, which was the determined cutoff value for cashew nut sIgE, was lower than those values identified in the literature. On the other side, limited studies on pistachio cutoff values predicting positive OFC were found. One of those studies was reported by Couch, with a negative predicted value of $50 \%$ of $11 \mathrm{~mm}$ for pistachio SPT. ${ }^{7}$ Ho et al. found this level was $\geq 6 \mathrm{~mm}$ with $86 \% \mathrm{PPV}$ and $64 \%$ NPV. ${ }^{22}$ In addition, Savvatianos $S$ et al. showed the pistachio sIgE of $0.35 \mathrm{kUA} / \mathrm{L}$ has a $96.8 \%$ sensitivity for the diagnosis of pistachio allergy. ${ }^{7}$ We found SPT and sIgE values for pistachio were $7.25 \mathrm{~mm}$ and $4.14 \mathrm{kUA} / \mathrm{L}$, respectively. Different from the previous studies, we could reach 95\% PPV neither cashew nut nor pistachio SPT and sIgE values. This may be due to the low frequency of pistachio and cashew nut allergic children, and not perform OFC test to all children. SPT values both for pistachio and cashew nut in our study and in the literature are very similar. Specific IgE values may be affected by high total IgE levels, and this is probably the reason why there are prominent differences among sIgE values between studies. According to the current study, SPT diameters and then the sIgE/Total IgE ratios were the most valuable parameters in predicting both the pistachio and cashew nut allergy may be due to for that possible reason. Therefore, more studies should be performed to clarify these particular differences in $\operatorname{sgE}$ values predicting allergy. 
In addition to optimal cutoff values based on Youden index, probability curves were generated for SPT, sIgE, and sIgE/Total IgE for both tree nuts. The study by Spergel et al., which also could not reach 95\% PPV for six major allergen foods other than tree nuts, indicated that sIgE levels for foods were clinically impractical in predicting food allergy. ${ }^{24} \mathrm{Al}$ though we could not reach $95 \%$ predictive value for pistachio sIgE or sIgE/Total IgE ratio, those curves can be useful for making OFC decision with cashew nut and pistachio.

It is well known that $\operatorname{sgE}$ may be falsely positive due to high total IgE levels. ${ }^{25}$ To eliminate the disparity in sIgE levels, and to standardize the values of sIgE among patients, we measured the ratio of sIgE/Total IgE. Further, we showed that this ratio was more valuable than sIgE alone. Gupta et al. studied sIgE/Total IgE ratio for predicting OFC passage. ${ }^{26} \mathrm{~A}$ higher ratio of $\operatorname{sigE} /$ Total $\operatorname{IgE}$ has been found in tree nut reactive patients compared to tolerant subjects. ${ }^{24}$ This ratio was more accurate than $\operatorname{sIgE}$ values alone for estimating the reactivity in OFC for tree nuts and peanut. ${ }^{26}$ We also observed that this ratio was elevated in reactive subjects and was better able to anticipate the clinical reactivity both for pistachio and cashew nut than values of sIgE alone.

Based on the data of the current study, we established a diagram resembling a decision tree guiding physicians to decide OFC. Combination of both SPT and sIgE values, which were above the cutoff values, would increase the possibility of positive reactions during OFCs than using only SPT value in predicting reactivity for both pistachio and cashew nut according to this diagram. Therefore, it would be better to use both of SPT and sIgE cutoff values before performing pistachio and cashew nut OFCs rather than only SPT-based management. We should also mention that those data and the created diagram need to be confirmed by further studies.

The most important limitation of this study was that OFCs were not performed on all patients, because informed consent for OFC could not be obtained from all parents/guardians. If all patients underwent provocation, the probability curves of pistachio sIgE and sIgE/Total IgE ratio may have reached 95\% accuracy, and cutoff values could achieve a higher PPV. The second limitation is that all OFC tests were open, not double-blind. To prevent false positivity, we continued OFC until objective symptoms were seen. The third important limitation is the small number of positive OFC, which may affect the accuracy of the probability curve. The strengths of the current study were that cutoff values, probability curves, clinical data of the patients, and laboratory parameters predicting OFC reactivity were all determined in same subjects. Children were selected randomly, regardless of clinical allergic reaction history, and there was no bias in the recruitment of the patients into the study.

\section{Conclusions}

Children who have had allergic reactions, particularly anaphylaxis, to cashew nut and pistachio in their history were more likely to have positive OFC with pistachio and cashew nut. SPT wheal size is the best parameter to estimate the reactivity for both pistachio and cashew nut. sIgE/Total IgE ratio followed by sIgE value are the other valuable tests for determining clinical reactivity. We propose the use of cutoff values and probability curves established for SPT, sIgE, and sIgE/Total IgE ratio, and the diagram while deciding for the proper time for OFC with pistachio and cashew nut.

\section{Acknowledgements}

The study protocol has been approved by the local ethical committee, and the parents/guardians gave written informed consent (Hacettepe University, GO 15/649-07).

\section{Author Disclosure Statement}

All authors declare that there is no conflict of interest. All authors declare that this research did not receive any specific grant from funding agencies in the public, commercial, or not-for-profit sectors.

\section{References}

1. Järvinen KM, Sicherer SH, Sampson HA, Nowak-Wegrzyn A. Use of multiple doses of epinephrine in food-induced anaphylaxis in children. J Allergy Clin Immunol. 2008;122:133-8.

2. Hsin YC, Hsin YC, Huang JL, Yeh KW. Clinical features of adult and pediatric anaphylaxis in Taiwan. Asian Pac J Allergy Immunol. 2011;29: 307-12.

3. USDA F. United States Department of Agriculture, Foreign Agricultural Service. Livestock and poultry: World Market and Trade 2014.

4. Weinberger T, Sicherer S. Current perspectives on tree nut allergy: a review. J Asthma Allergy. 2018;11:41-51.

5. Muraro A, Werfel T, Hoffmann-Sommergruber K, Roberts G, Beyer K, Bindslev-Jensen C, et al. EAACI food allergy and anaphylaxis guidelines: diagnosis and management of food allergy. Allergy. 2014;69:1008-25.

6. Grigg A, Hanson C, Davis CM. Cashew allergy compared to peanut allergy in a US tertiary care center. Pediatr Asthma Allergy Immunol. 2009; 22:122-9.

7. Couch C, Franxman T, Greenhawt M. Characteristics of tree nut challenges in tree nut allergic and tree nut sensitized individuals. Ann Allergy Asthma Immunol. 2017;118:591-6.

8. Savvatianos S, Konstantinopoulos AP, Borgå Å, Stavroulakis G, Lidholm J, Borres MP, et al. Sensitization to cashew nut $2 \mathrm{~S}$ albumin, Ana o 3, is highly predictive of cashew and pistachio allergy in Greek children. J Allergy Clin Immunol. 2015;136:192-4.

9. Muraro A, Roberts G, Worm M, Bilò M, Brockow K, Fernández Rivas M, et al. Anaphylaxis: guidelines from the European academy of allergy and clinical immunology. Allergy. 2014;69:1026-45.

10. Eichenfield LF, Tom WL, Chamlin SL, Feldman SR, Hanifin JM, Simpson EL, et al. Guidelines of care for the management ofáatopicádermatitis: Section 1. Diagnosis and assessment of atopic dermatitis. J Am Acad Dermatol. 2014;70:338-51.

11. Pedersen SE, Hurd SS, Lemanske RF, Becker A, Zar HJ, Sly PD, et al. Global strategy for the diagnosis and management of asthma in children 5 years and younger. Pediatr Pulmonol. 2011;46:1-17.

12. Bateman ED, Hurd S, Barnes P, Bousquet J, Drazen J, FitzGerald M, et al. Global strategy for asthma management and prevention: GINA executive summary. Eur Respir J. 2008;31:143-78.

13. Brożek JL, Bousquet J, Agache I, Agarwal A, Bachert C, Bosnic-Anticevich S, et al. Allergic Rhinitis and its Impact on Asthma (ARIA) guidelines-2016 revision. J Allergy Clin Immunol. 2017;140:950-8.

14. Niggemann B, Lange L, Finger A, Ziegert M, Müller V, Beyer K. Accurate oral food challenge requires a cumulative dose on a subsequent day. J Allergy Clin Immunol. 2012;130:261-3.

15. Simons FER, Ebisawa M, Sanchez-Borges M, Thong BY, Worm M, Tanno LK, et al. 2015 update of the evidence base: World Allergy Organization anaphylaxis guidelines. World Allergy Organ J. 2015;8:32-48.

16. Kattan JD. Oral food challenges in routine clinical practice. Curr Allergy Clin Immunol. 2013;26:122-9.

17. Sicherer SH, Sampson HA. Food allergy. J Allergy Clin Immunol. 2010;125:116-25. 
18. Clark A, Ewan P. Interpretation of tests for nut allergy in one thousand patients, in relation to allergy or tolerance. Clin Exp Allergy. 2003;33: 1041-5.

19. Stiefel G, Anagnostou K, Boyle R, Brathwaite N, Ewan P, Fox A, et al. BSACI guideline for the diagnosis and management of peanut and tree nut allergy. Clin Exp Allergy. 2017;47:719-39.

20. Wananukul S, Chatchatee P, Chatproedprai S. Food induced urticaria in children. Asian Pac J Allergy Immunol. 2005;23:175-9.

21. Ludman S, Ballabeni P, Eigenmann PA, Wassenberg J. Predicting positive food challenges in children sensitised to peanuts/tree nuts. Pediatr Allergy Immunol. 2013;24:276-81.

22. Ho MH, Heine RG, Wong W, Hill DJ. Diagnostic accuracy of skin prick testing in children with tree nut allergy. J Allergy Clin Immunol. 2006;117:1506-8.
23. Inoue $\mathrm{T}$, Ogura $\mathrm{K}$, Takahashi $\mathrm{K}$, Nishino $\mathrm{M}$, Asaumi T, Yanagida N, et al Risk Factors and Clinical Features in Cashew Nut Oral Food Challenges. Int Arch Allergy Immunol. 2018;175:99-106.

24. Spergel JM, Boguniewicz M, Schneider L, Hanifin JM, Paller AS, Eichenfield LF. Food allergy in infants with atopic dermatitis: limitations of food-specific IgE measurements. Pediatrics. 2015;136:e1-11.

25. Horimukai K, Hayashi K, Tsumura Y, Nomura I, Narita M, Ohya Y,et al. Total serum IgE level influences oral food challenge tests for IgE-mediated food allergies. Allergy. 2015;70:334-7.

26. Gupta RS, Lau CH, Hamilton RG, Donnell A, Newhall KK. Predicting Outcomes of Oral Food Challenges by Using the Allergen-Specific IgE-Total IgE Ratio. J Allergy Clin Immunol Pract. 2014;2:300-5. 